\title{
DIE ERSTELLUNG EINES LERNMEDIUMS FÜR DEUTSCHE GESCHICHTE
}

\author{
Windy Br Manullang \\ Laurensius Tampubolon \\ Risnovita Sari
}

\begin{abstract}
AUSZUG
Das Ziel dieser Untersuchung ist es, um eine Lernmedium mit der Wondershare Filmora Software zum Thema, Der Erste Weltkrieg, der Zweite Weltkrieg, Kalten Krieg und Deutsche Bedigungen" zu erstellen. Die Erstellungsuntersuchung werden in dieser Untersuchung angewendet. Der Prozess der Erstellung des Lernmediums mit der Wondershare Filmora verwendet ADDIE - Model. Die Erstellungsphase besteht aus fünf Phasen. Die Phasen sind (a) Analyse, (b) Design, (c) Entwicklung, (d) Implementation und (e) die Evaluation. In dieser Untersuchung wird ein neues Lernmedium erstellt. Die Daten dieser Untersuchung sind information über „,Der Erste Weltkrieg, der Zweite Weltkrieg, Kalten Krieg und Deutsche Bedigungen" bei der Deutschen Geschichte. Die Datenquelle dieser Untersuchung kommt aus dem Youtube und aus dem Artikel. Diese Untersuchung wird in der Bibliothek an der Fakultät für Sprachen und Kunst an der Staatlichen Universität von Medan durchgeführt. Die Ergebnisse der Erstellung eines Lernmediums mit Wondershare Filmora zum Thema ,Der Erste Weltkrieg, der Zweite Weltkrieg, Kalten Krieg und Deutsche Bedigungen", ist es das Video und die Übungen. Nach der Erstellung wurde das Lernmedium bei der Experten validiert oder überpruft, Basierend auf der Validierung ist das Lernmedium gut.
\end{abstract}

Schlüsselwörter : Die Erstellung, Lernmedium, Deutsche Geschichte

\section{EINLEITUNG}

Das Lernen ist ein Komplexer Prozess, der jedem passiert und es dauert ein Leben lang. Eine Veränderung der Verhaltens bei ihm selbst ist der Zeichen der Mensch gelernt hat. Gagne (1991:160).

Das Wort Medien entstammt aus dem Lateinischen und das Wort Medien ist der Plural von dem Wort, das als vermitten bedeutet. Mcgraw - Hill (1959:3) behauptet, In discussion of media, another phrase that represents a stage in the evolution of knowledge about teaching is "Instructional technology goes beyond any particular medium or device. In this sense, instructional technology is more than the sum of its parts. This definition Implies that, for Improvement of 
instruction, systematic planning and the wise and skillfull use the products of technology are basic.

Hamalik (im Arsyad 2012:15) erklärt, die Verwendung von Medien beim Lernprozess kann die Motivation steigern und bei Schülern einem Psychologischen Effekt haben, der Lernen stimuliert. Das Medium, das benutzt wird, ist ein audiovisuelles Medium oder Video. Der Begriff „Video" nach Kamus Besar Bahasa Indonesia (KBBI), bezeichnet eine Aufnahme von Live Bilder oder Fernsehprogrammen die auf einem Fernsehgerät ausgestrahlt werden, oder mit anderen Worten Videos sind bewegte Bilder mit Ton.

Die Vorteile der Verwendung von "Videos" sind folgende : mit der Hilfe „Video" können die Stundenten ihr Höverstehen steigern. Das Medium „Video" kann beweten, was haben die Stundenten gehört, das Medium „Video” kann auch denken über Unterricht. Video - Medien können nicht von der Beteiligung von Hardware und Software getrennt werden. Die Software, die benutzt wird ist, Wondershare Filmora.

Das Lernen von deutschen Geschichte zielt darauf ab, das Wissen über die deutsche Geschichte zu erklären, das Wissen über die Kultur, die Gesellschaft und die Entwicklung der deutschen Staaten heute ist. Das Material eines Semester umfasst unter anderem die Geschichte von 16 Staaten, den ersten Weltkrieg, Weimarer Republik, den zweiten Weltkrieg, Teilung des deutschen Staates, die Ära des Kalten Kriegs von 30 Jahren, die Wiedervereinigung Deutschlands und die neuesten Deutschen bedingungen.

Aufgrund der Lernerfahrung der Forscherin, der Unterricht der deutschen Geschichte verwendet traditionelle Lernmedien, das Methode ist nicht interessant und noch nie effektiv. Die Forscherin beschränkt das zu diskutierende Material in dieser Forschung auf die Geschichte des ersten Weltkriegs, zweiten Weltkrieg, die Ära des Kalten Kriegs von 30 Jahren und die neuesten deutschen Bedingungen.

Mit dem oben genannten Material sind die Forscher daran interessiert, eine Video - Geschichte in etwas Interessantes, Konzeptionelles, voller Sinn und Qualität zu machen, um die Motivation der Schüler zu generieren. Deshalb wollen 
die Forscher mit dem Titel “ der Erstellung des Lernmediums für Deutsche Geschichte" Forschen um das Problem zu lösen.

\section{THEORETISCHE GRUNDLAGE}

\section{Begriff der Erstellung}

Sugiyono (2016:407) erklärt, Entwicklung verwendet eine Methode, um ein bestimmtes Produkt zu erhalten und testet die Wiksamkeit des Produkts. So kann man schliessen, dass etwas entwickelt wird, was noch nicht existiert hat oder etwas, das bereits existiert wird noch besser gemacht.

\section{Das Erstellungsmodell}

Es gibt verschiedene Modelle, um ein Medium zu entwickeln. Das ADDIE Model ist eine davon. Dieses Modell ist die Theorie von Reiser und Mollenta. Reiser und Mollenta in Rohman und Amri (2013:210) beschreiben, dass das ADDIE Modell fünf Phasen, darunter sind : 1) Analyse, 2) Design/Konzeption, 3) Development / Entwicklung, 4) Implementation / Umsetzung, 5) Evaluation.

\section{Der Begriff der Lernmedien}

Nach Meinung von Gagne' \& Briggs ist das Lernmedium ein Werkzeug, den Inhalt der Unterrichtsmaterialien $\mathrm{zu}$ verwenden, um Inhalte aus Buch, Kassette, Film, Foto, Bild, Fernsehen, Computer, und und andere zu vermitteln (vgl. Arsyad 2013:4).

Rossie und breidle (in Sanjaya, 2008:58) erklären, dass die Lernmedien alle Kommunikationsmittel für die Ausbildung sind. Beispielweise sind Radio, Fernsehen, Bücher, Zeitung, und Magazine.

\section{Arten von Lernmedien}

Seels und Glasgow stellen dar, dass es solgende Arten von Lernmedien gibt, die beim Lesrnprozess verwendet werden können. Diese sind: (1) visuelles Medium, (2) audiomedium, (3) audiovisuelles Medium, (4) Klassen von Mediumpräsentation: (a) die erste Klasse: grafiches und gedrucktes Material, (b) die zweite Gruppe: das Medium-Projektionen, (c) die dritte Gruppe; Audio Medium; (d) die vierte Gruppe; visuelles Medium, (e) fünfte Gruppe; Film, (f) 
sechste Gruppe; Fernsehenmedium, (g) siebte Medium; Multimedium, und (h) achte Medium; interaktives Medium und dreidimensionales Medium.(vgl. Arsyad 2013: 35)

\section{Der Begriff Video}

Sianipar (in Andi 2014:2) definiert Videos oder bewegte Bilder als eine Reihe von vielen frames (Rahmen). Jeder Frame ist die Aufnahme einer stufenweisen Bewegung. Die Augen können den Unterschied (die Pause Punkt Übertragung) zwischen den Frames nicht erkennen, wenn die Bilderserien mit einer Geschwindigkeiten von über 20 Frames pro Sekunde gedreht werden, sodass das Gehirn die Illusion einer Bewegung wahrnimmt.

Andi (2014:3) erklärt, dass ein Video eine Reihe von animierten Clip ist, Audiodateien und Bilddateien die erstellt Animationen dann bearbeitet und die Wirkung geben. Ayuningrum (in Andi 2015:21) dagegen zeigt, dass Videos Audio - visuallen Materialien sind, welche Nachrichten/ Liefern und ald Lernmaterialien, welche in einer Videokassette verpackt und an einen TV Monitor angeschlossen sind.

\section{Der Prozess der Erstellung von Video}

Youtube ist eine Sozielmedien, die Forscherin verwendet die Anwendung für uploaden das Video deutschen Geschichte. Die Forscherin, machen das Video mit App Wondershare Filmora. In Wondershare Filmora man kann editieren das Video, design background, Ton, usw.

\section{Konzeptuelle Grundlage}

Durch die Korrelation der Eigenschaften der Deutschunterrichts mit der Erstellung von Wissenschaft und Technik und den Anforderungen der Erstellung von Bildung, sind Audiovisuelle Medien als Werkzeug der Unterrichtsgestalltung sehr geeignet. Sie unterstützen die Lehrer sowie das Lernen der Schüler. Somit würde der Deutschunterricht für Studenten interessanter werden. In der Erstellung eines Lernmediums für Deutsche Geschichte zum themen “der Erste Weltkrieg, der Zweite Weltkrieg, Kalten Krieg und die neuesten das Heutige Deutschland" wird mit Hilfe des App Wondershare Filmora. 
In dieser Untersuchung wird das ADDIE Modell durchgeführt. Es gibt fünf Phasen, sind : 1. Analyse, 2. Design/Konzeption,

3. Development/Entwicklung, 4. Implementation/Umsetzung, 5. Evaluation. Mit dieser Medien - Video wird gehofft, dass die deutschstudenten interessanten für deutsche Geschichte unterrichten. Inzwischen die Qualität zu testen, diese medienbasierte Video, werden für die weitere Überarbeitung Phase des Produkts bewertet die Machbarkeit dieses Lernmedienprodukte zu bestimmen.

\section{UNTERSUCHUNGSMETODE}

Diese Untersuchung ist eine Erstellungsuntersuchung zur Erstellung eines Lernmediums für deutsche Geschichte.

Diese Untersuchung wird in der Digitalbibliothek an der staatlichen Universität Medan und in dem kleinen Sprachlesenraum in der Fakultät für Sprache und Kunst durchgeführt. Diese Untersuchung benutzt die Theorie von ADDIE. Die Erklärung dieser Theorie wurde schon im Kapitel II gegeben. Tegeh (2014:78) schreibt, dass der Entwicklungsprozess bei der Erstellung von Lehrangeboten auf Basis des ADDIE Modells in fünf Phasen untergliedert wird. Diese sind Analyse, Design/Konzeption, Development/ Erstellung, Implementation/Umsetzung,und Evaluation .

\section{ERGEBNISS DER UNTERSUCHUNG}

Dieses Kapitel handelt von den Untersuchungsergebnissen. In diesem Kapitel werden die Ergebnisse der Erstelllung des Lernmediums mit der Wondershare Filmora software zum Thema „,Der erste Weltkrieg, zweite Weltkrieg, 30 Jahre Kalten Krieg, und das Heutige Deutschland aus dem Youtube erklärt. Jeder Schritt hat ein eigenes Untersuchungsergebnis. Die Schritte der Untersuchung und die Ergebnisse werden in diesem Kapitel erklärt.

\section{Der Prozess der Erstellung}

In dieser Untersuchung wird die Theorie des ADDIE - Modells von Reiser und Mollenda benutzt. Diese Theorie besteht aus fünf Phasen, 
nämlich : Analyse, Design/Konzeption, Entwicklung, Implementation, und Evaluation. In diesem Unterkapitel wird jede Phase mit ihrem Ergebniss erklärt.

\section{Analyse}

Diese ist die erste Phase, um ein Lernmedium für Deutsche Geschichte zu erstellen. In dieser Phase der Beobachtung werden Information über das Lernen der Deutschlernenden, ihre Bedürfnisse und die Lernmedien im Deutschgeschichteunterricht gesammelt. Zunächst wird eine Umfrage gemacht. Das Ergebnis der Umfrage war, die Bedürfnisse den Deutschlernenden ist, was die Medien nicht finden, dass die Deutschlernenden eines Lernmediums für Deutsche Geschichte brauchen. Deshalb ist es sehr wichtig, ein Lernmedium zu erstellen.

\section{Design}

Zweite Phase ist die nächste Erklärung von der ersten Phase. Zuerst wird die Geschichte „Erste Weltkrieg, Zweite Weltkrieg, Kalten Krieg und das Heutige Deutschland" entworfen, die wörter gewählt und die Satze geschrieben. Danach werden die Video Geschichte gewählt. In dieser Phase wird die Applikation Wondershare Filmora erstellt. Das Materien über der Erste Weltkrieg, der Zweite Weltkrieg, 30 Jahren Kalter Krieg, und das Heutige Deutschland sind aus dem Buch und Youtube. In dieser Phase werden viele Video aus verschiedenen Quellen gesammelt. Dann wird das Video basierend auf dem Thema geordnet, das gemacht wird. Dann ist es basierend auf dem Thema geordnet, dann wird der Prozess der Videobearbeitung begonnen. Von der Erstellung von Untertitel, HD Einstellungen, Effekten und Übungen am Ende des Video.

\section{Der Ergebniss des Video}

Das Ergebnis der Untersuchung ist eine Video mit Wondershare Filmora Applikation . Diese Video dauert 3 bis 11 minuten und die Datei umfasst 925 MB (Megabyte). Die Video kann mithilfe des GOM Players und VLC geöffnet 
werden. Unten werden einige der Ergebnisse des Designs des Lernmediums erklärt.

\section{Diskussion}

Basierend auf den Ergebnissen über des Lernmediums, das mit der Wonder Share Filmora wurde zusammengefasst. In dieser Untersuchungs wird die ADDIE Modell Theorie benutzt. Diese Theorie besteht aus fünf Stufen, nämlich: (1) Analyse, (2) Design/Konzept, (3) Entwicklung, (4) Implementation, (5) Evaluation. In diesem Kapitel wird jede Stufe mit ihrem ergebnisse erklärt. In der Ermittlungsphase wird zuerst die Datensammlung und die Problemidentifizierung durchgeführt.

In dieser zweiten Phase wird das Konzept/Design des Lernmediums mit Video entworfen, um ein interessantes Lernmedium zu erstellen. Das Design wird an das Thema ,der Erste Weltkrieg, der Zweite Weltkrieg, Kalter Krieg, und das Heutige Deutschland " angepasst, die in derVideo integriert werden, Dieses Video bestehen aus 4 Thema und 4 Video. Video bewegte Bilder erzählen über die Erste Weltkrieg, Der Zweite Weltkrieg, Kalter Krieg und das Heutige Deutschland, der in das Dorf zurückkehren möchte. JedesVideo erzähltüber die Weltkrieg und die Geschichte. Die nächste Phase ist Entwicklungphase. Das Lernmaterial wird mit den Video entwickelt. Das Lernmedium wurde schon mit Tone, Bildern, Subtittle, Animierten und Backsound, so dass es interessantes Medium enthält.

In der vierten Phase ist das Implementation. Der Expert hat das Lernmedium mit dem Video analiysiert und korrigiert. Danach wird in der Implementation das erstellte Lernmedium mit demVideo validiert. Dann in der fünf Phase ist Evaluation. Der Expert des Lernmediums benotet: von 16 Kategorien, sodass die Note Sie dass Lernmedium 82.81(gut) ist.

Basierend auf der obigen Erklärung kann zusammengefasst werden, dass die Erstellung des Lernmediums mit der Video zum Thema "die Erste Weltkrieg, der Zweite Weltkrieg, Kalter Krieg und das Heutige Deutschland" aus dem Youtube mit der ADDIE Theorie sehr gut ist und Lehrern können das Lenmedium in der Klasse benutzt. 
Studia: Journal des Deutschsprogramms p-ISSN 2301-6108 e-ISSN 2654-9573

https://jurnal.unimed.ac.id/2012/index.php/studia/index

\section{SCHLUSSFOLGERUNG}

Nach den Untersuchungsergebnissen fallen die Schlussfolgerungen folgendermaßen aus:

1. Der Prozess der Erstellung eines Lernmediums mit Video für die Steigerung der Deutsche Geschichte besteht aus der Erklärung der Phasen von ADDIE. Diese sind: (1) Analyse, (2) Design/Konzeption, (3) Entwicklung, (4) Implementation und (5) Evaluation.

2. Das Ergebnis der Erstellung eines Lernmediums mit Video für die Steigerung der Deutsche Geschichte sind:

a. In dieser Untersuchung wurden 4 Video erstellt.

b. Dieses Videobestehen aus 4 Thema und kann klingen.

c. Die Video mit dem Thema 'Der Erste Weltkrieg, der Zweite Weltkrieg, Kalten Krieg, und Deutschen Bedigungen" haben von der Experten des Designs evaluiert. Danach wird die Evaluation der Wörter und der Sprache der Video korrigiert, die von dem Muttersprachler gemacht wird.

d. Der Expert des Lernmediums benotet: von 16 Kategorien, sodass die Note Sie dass Lernmedium 82.81 (gut) ist. Basierend auf der Meinungen der Experten sind die Video gut und inhaltlich ist das Thema sehr interessant und das Ziel wird deutlich

\section{LITERATURVERZEICHNIS}

Arsyad,Azhar. 2002. Media Pembelajaran. Jakarta : PT Raja Grafindo Persada.

Benecke, W. Dieter. 1998. Kleine Geschichte der Deutschen. Saleh,Ridwan.

Goethe - Institute. Bonn: INTER NATIONES.

Djamarah dan Zain. 2013. Strategi Belajar Mengajar. Jakarta: Rineka Cipta.

Komputer, Wahana dan Andi. 2015. Video Iklan Komersial. Yogyakarta: ANDI.

Ojong, P.K. 2002. Perang Eropa. Jakarta: Buku Kompas.

Pratowo,Andi. 2014. Pengembangan Bahan Ajar Tematik. Jakarta:Kencana.

Sanjaya,Wina. 2008. Strategi Pembelajaran. Jakarta: Kencana.

Sanjaya,Wina. 2008. Pencanaan dan Desain Sistem Pembelajaran. Jakarta: Kencana. 
Studia: Journal des Deutschsprogramms p-ISSN 2301-6108 e-ISSN 2654-9573

https://jurnal.unimed.ac.id/2012/index.php/studia/index

Sjamsuddin,Helius. 2012. Metodologi Sejarah. Yogyakarta: Ombak.

Siboro,Julius. 2012. Sejarah Eropa. Yogyakarta: Ombak.

https://www.klikmania.net/aplikasi-edit-video/wird am 11 mei 2017/ um 23:30 Uhr gelesen.

https://www.youtube.com/watch?v=jChIuwE4Tc/wird am 12 mei 2017/um 01:24 Uhr anschauen.

https://www.youtube.com/watch?v=2alq4Uk/wird am 12 mei 2017/um 01:18 Uhr anschauen.

https://www.youtube.com/watch?v=Dbo0vIy67w8, wird am 12 mei 2017/um 02:01 Uhr anschauen.

http://thesimpleclub.de/abi2018, wird am 25 oktober 2017/um 23:00 Uhr anschaun.

http://www.youtube.com/thesimplehistory, wird am 25 oktober 2017/um 23:29 Uhr anschauen.

http://www.thesimpleclub.com, wird am 25 oktober 2017/um 01:09 Uhr anschauen.

www.simpleshow.de, wird am 26 oktober 2017/ um 19:45 uhr gelesen.

http://www.youtube.com/topzehn, wird am 26 oktober 2017/ um 21:12 Uhr anschauen. 